\title{
Institutional Factors Affecting Retention of HIV Patient on Treatment at Wuse General Hospital, Abuja, Nigeria
}

\author{
Uba Sabo Ado ${ }^{1 *}$, Thaddeus Olatunbosun ${ }^{2}$, Adeyemi Adedayo ${ }^{3}$, Uba Belinda Vernyuy ${ }^{4}$, \\ Babashani Musa ${ }^{5}$ \\ ${ }^{1}$ Department of Public Health, Federal Ministry of Health, Abuja Nigeria \\ ${ }^{2}$ APIN Public Health Initiatives, Abuja Nigeria \\ ${ }^{3}$ Department of Public Health, Centre for Infectious Diseases Research and Evaluation, \\ Lafia, Nigeria \\ ${ }^{4}$ African Field Epidemiology Network, Asokoro Abuja, Nigeria \\ ${ }^{5}$ Department of Medicine, Aminu Kano Teaching Hospital, Kano Nigeria
}

\begin{abstract}
Retention on treatment is a critical factor in reducing HIV-related morbidity and mortality, the incidence of new infections, and development of drug resistance among children and adults. This study examined institutional factors affecting retention of HIV patients on treatment, care, and support services at Wuse General Hospital, Abuja. A retrospective cohort study was used to access institutional factors that influence HIV patient retention on treatment. About 4,564 HIV patients were ever enrolled on a treatment programme, and 2,791 patients are currently accessing treatment. A total of 240 clients were recruited for the study, and a pre-tested interviewer-administered semistructured questionnaire was used for data collection. Data were entered into Stata/MP 15.0 and then exported into SPSS version 23.0 for statistical analysis of the 240 respondents interviewed, the main institutional factor affecting retentions were long waiting time (61.7\%), high patient load (46.9\%), inadequate space for clinics (48.3\%), Poor quality of services (40.8\%), difficulty in accessing health facility (32.9\%) and inadequate health workers/staff (31.3\%). A review of socio-demographic characteristics of respondents and institutional related factors affecting retention in treatment showed an association between marital status $(p=0.000)$, religion $(p=0.006)$, employment status $(p=0.009)$, and institutional related factors affecting retention in care. Retention of HIV patients in treatment and care support services is a major determinant in achieving sustained viral suppression and reduced community HIV transmission. Therefore, strategies to mitigate institutional barriers to treatment retention should be promoted to optimize retention of HIV patients in care.
\end{abstract}

Keywords: HIV/AIDS, Institutional Factors, Retention, Treatment.

\section{Introduction}

HIV/AIDS is a public health problem, and one of the leading causes of illness and death in adults globally [1]. Antiretroviral Therapy (ART) is the gold standard for the management of HIV infection, and optimal ART leads to rapid improvement of clinical indices and makes PLHV become less susceptible to new infections [2]. With the recent scale-up in the national HIV programme and improved access to ART, retention of People Living with HIV (PLHIV) in treatment and care is becoming a major concern. Effective ART decreases the risk of transmitting the HIV virus from one person to another $[3,4]$ and improves the quality of life for PLHIV.

Patients lost to follow-up (LTFU) at different stages of the HIV cascade may increase HIV 
transmission, mortality, and morbidity rates as well as hinder efforts to control the HIV epidemic [5, 6].

Linkage to care after testing positive to HIV is not enough to maximize the benefits of HIV services as one needs to be retained in care in order to fully benefit from antiretroviral therapy. Patient retention in the HIV treatment cascade has become a challenge over time, with high rates of loss to follow up. They are lost at every step along the continuum of care, particularly from the time of HIV diagnosis, through initiation in ART, and during treatment. It is recognized that poor retention of patients in care and treatment is a major driver of poor performance and increased morbidity and mortality in HIV/AIDS programmes [7, 8]. Poor retention in care has been associated with higher mortality for patients in both highincome and resource-limited settings [9, 10]. Retention in care is a key indicator of ART programmes' effectiveness and is pivotal for achieving the Global 90-90-90 targets for ending the HIV AIDS epidemic [11]. HIV retention and adherence are associated with viral load suppression, reduction in HIV transmission, a decline in cases of HIV drug resistance, and disease-associated morbidity and mortality [12]. For cost-effectiveness, retention is a critical issue for ART programs. As programs are trying to maximize the limited resources available to combat the impact of HIV infection and increase value for money, a failure to ensure good retention of PLHIV in care is costly for both the program and the patients themselves.

Patients who are lost to follow up (LTFU) experience higher mortality than those remaining in care $[13,14,15]$. In previous studies, patients traced after loss to follow-up (LTFU) reported higher rates of mortality compared to those retained in care [16]. Identifying the factors associated with the risk of loss to follow-up is necessary to inform strategies that promote retention in care.
Despite the benefits of retention in care, it is challenging for the health care facilities (HCFs) to keep all patients in HIV care [17]. Some health facility factors that affect retention of PLHIV in care have previously been identified. Long waiting time at facilities has been documented as a contributing factor to low retention rates $[18,19,20]$. Other facility-level factors that can affect LTFU include the location of the healthcare, staffing characteristics, and the role of patient-provider relationships [21, 22]. The institutional factors that were examined in this study include long waiting, high patient load, stock of out of ARVs, and travel distance to ART centres/transportation fare. Other factors include inadequate space for ART clinics, inadequate health workers/staff, poor quality of services, and difficulty in accessing health facilities.

\section{Materials and Methods}

A retrospective cohort study was employed to access factors affecting PLHIV retention in ART. An in-depth interview using semistructured questionnaires was used to extract patient information on economic factors affecting retention of PLHIV in care and treatment. The interview was conducted with the consent of participants, and adequate privacy was maintained to ensure maximum cooperation.

\section{Study Area}

This study was carried out in Wuse General Hospital, Abuja, Nigeria - a secondary health facility that is one of the ART sites in the Federal Capital Territory (FCT). It offers comprehensive HIV care services, including antiretroviral therapy (ART). The decision to choose this institution was because the hospital caters for a huge number of people on treatment for HIV and AIDS within Abuja town and its environs and has the required standard and capacity to offer optimal services. About 4,564 
PLHIV patients have been enrolled on the ART programme in Wuse General Hospital since the inception of the ART programme in 2005. About 2,791 patients are currently accessing treatment as of June 2020 . The facility provides comprehensive HIV services including, CD4 count, viral load, haematology and chemistry, adherence counseling, preventive services, HIV testing service (HTS), management of opportunistic infection (OIs), and adverse drug reactions. Patients who are infected with HIV are diagnosed at various counseling and test points in the hospital and are referred to ART clinics within the hospital for commencement of treatment as recommended in the Nigerian National Treatment Guidelines. ART adherence counseling is offered at the initiation of treatment and subsequently through group counseling or individual counseling by trained adherence counsellors. During the adherence counseling session, patients' commitments and treatment adherence are emphasized before they commenced ART to optimize retention.

\section{Study Design}

A retrospective cohort study design was used to access patient factors, health systems, social and economic factors that influence PLHIV retention on ART in Wuse General Hospital, Abuja, Nigeria.

\section{Target Population}

The study targeted PLHIV that are 18 years and above who were retained on care for at least 12 months since the inception of the ART programme in 2005 till June 2020.

\section{Inclusion Criteria}

To qualify as participants in this study, you must be diagnosed and confirmed to have HIV infection and must have been in care for at least 12 months. Participants also must be mentally competent, capable of communicating, and willing to participate (consented), and must be 18 years or above.

\section{Exclusion Criteria}

PLHIV who do not consent for the study and mentally deranged person, those who have been receiving treatment for less than 12 months, and those incapable of communicating were excluded.

\section{Sample Size Determination}

The sample size for the study was calculated using the formula below:

$$
n=\frac{\left(Z \alpha^{2} x P x(1-P)\right.}{d^{2}}
$$

$\mathrm{n}=$ the sample size

$\mathrm{Z} \alpha=$ standard error deviate at $95 \%$ confidence level $=1.962$

$\mathrm{p}=$ The percentage of patients retained at 12 months was $81.2 \%$ (i.e., $\mathrm{p}=0.812$ ) from a study on outcomes of Nigeria's HIV/AIDS treatment programme for patient-initiated on ART 2004-2012 [23].

$\mathrm{q}=1-\mathrm{P}$

$\mathrm{d}=$ Precision $=5 \%$

$$
n=\frac{1.96^{2} \mathrm{x} \times(1-\mathrm{P})}{0.05^{2}}
$$

$n=\frac{1.962 \times 1.962 \times(0.812) \times(0.188)=0.5876 / 0.05^{2}}{0.05^{2}}$

Minimum sample size $(n)=235.04$.

For a Population of less than 10, 000.

The formula $\mathrm{n} /(1+\mathrm{n} / \mathrm{N})$ was used in the determination of the minimum sample size required.

Where:

$\mathrm{N}$ is the estimated study population that is less than 10,000, which is 3000 (obtained from the facility records).

$\mathrm{n}$ is the minimum sample size required for a population greater than 10,000 .

Therefore,

$235 /(1+235 / 3000)$

$235 / 1.078$

$=218$

$10 \%$ of non-response rate of 22 was added, making a total of 240 . 


\section{Sampling Technique}

A systematic random sampling technique was used to select participants for the study. The sampling units included PLHIV age 18 years and above who were retained on ART for at least 12 months prior to the study, including those that were lost to follow up who were contacted through phone calls for a scheduled appointment. From the list of patients attending each clinic day, PLHIV who met the inclusion criteria were consecutively recruited from the ART clinic into the study till the desired sample size was attained. Averagely, a total of $100 \mathrm{HIV}$ clients attends the clinic per day; a total of 24 eligible patients was recruited per clinic day for 10 clinic days until the required sample size of 240 was achieved.

\section{Data collection}

A pre-tested interviewer-administered semistructured questionnaire was used to collect data for this study. The semi-structured interviews allowed the study participants to give detailed information on the reasons why HIV and AIDS patients were not retained in treatment or lost to follow-up. The study instruments consist of sections on sociodemographic data, social and economic factors, drug history (side effects and adverse drug reactions), health system factor and patientrelated factors that influenced retention, stigma, cost of transportation, accessibility and availability of services, family and social support and mental health problems. The study participants were educated about the study, its benefits, and their consent was obtained before commencement of the study, and participants were assured of the confidentiality of their information.

\section{Data analysis}

There was a general review of filled questionnaires, and data were cleaned, coded, and entered into Stata/MP 15.0 (Stata Corp LLC, Texas, United States). Data cleaning and complete checks of entered data were done to exclude incomplete, inaccurate, and doubleentry before analysis. Electronic data backup was done using a flash drive. The data was then exported into SPSS version 23.0 (SPSS 2018 IBM Corp, New York, United States) for statistical analysis. Data were summarized using tables for key elements. Results were presented in tables for frequencies and measures of central tendencies with the appropriate measure of dispersion. Univariate analysis was performed, showing frequencies, proportions, and mean. Bivariate analyses for associations between socio-demographic factors, patient or individual factors, social factors, economic and health institution factors, and retention were performed. The bivariate analysis included the use of the $\mathrm{Chi}^{2}\left(\chi^{2}\right)$ test or Fisher's exact test for statistical significance. Multivariate analysis using binary logistic regression was used to identify independent predictors that affect retention of PLHIV on treatment. A p-value $<0.05$ was considered to be significance.

\section{Calculation of Retention in Care}

In this study, there are 4,564 PLHIV that ever enrolled in the ART programme in Wuse General Hospital since the programme was established in 2005. Currently, 2,791 patients are retained in care and accessing treatment as of June 30, 2020, prior to data collection. The number of attritions was 1,773 obtained from the hospital Electronic Medical Record (EMR) system, which includes patients who were lost to follow-up, dead, relocation, and transferred out. The retention rate was calculated as currently retained in treatment divided by the number of PLHIV ever enrolled in the ART programme.

The retention rate (\%) was computed as follows:

Retention rate $(\%)=$ Retention in care/ (retention in care + attrition) $* 100$ [24].

Retention Rate $=2,791 /(2,791+1773)$

$=2,791 / 4,564$

$=61.1 \%$ 


\section{Ethical Consideration}

Ethical approval was sought from the ethical review committee from Federal Capital Territory (FCT) and Wuse General Hospital, Abuja, before the commencement of the study. Consent was obtained from participants before administering the questionnaire using an informed consent form. Clients that declined interviews were not coarse to participate in the study. The confidentiality and privacy of the participants were maintained throughout the process of the interview. Information collected was not linked to the participants because the questionnaire does not have provision for participant's names and other identifying details that may be linked to the participants, including hospital numbers.

\section{Limitations of the Study}

This study was conducted in Wuse General Hospital, Abuja, which is located in an urban area of Federal Capital Territory Abuja, and this might have excluded a huge number of HIV patients from rural areas who may have different factors that affects their retention in care; hence, the study may not be applicable to the rural settings.

\section{Results}

A total of 240 participants were enrolled in the study. Table 1 below presents the summary of the socio-demographic characteristics of the study participants.

Table 1. Socio-demographic Characteristics of Respondents

\begin{tabular}{|l|l|l|}
\hline Characteristics & Frequency & Percentage \\
\hline Age & 74 & 30.8 \\
\hline $20-29$ & 92 & 38.3 \\
\hline $30-39$ & 57 & 23.8 \\
\hline $40-49$ & 13 & 5.4 \\
\hline $50-59$ & 4 & 1.7 \\
\hline $60-69$ & \multicolumn{2}{|l|}{$34.4 \pm 10.2$} \\
\hline Mean & 64 & 26.7 \\
\hline Sex & 176 & 73.3 \\
\hline Male & \multicolumn{2}{|l|}{} \\
\hline Female & 37 & 15.4 \\
\hline Education & 42 & 17.5 \\
\hline No formal education & 37 & 37.1 \\
\hline Primary & 89 & 30.0 \\
\hline Secondary & 72 & 39.2 \\
\hline Tertiary & 94 & 43.8 \\
\hline Marital status & 105 & 8.3 \\
\hline Never married & 20 & 8.8 \\
\hline Married & 21 & 58.8 \\
\hline Separated/Divorced & 141 & 11.7 \\
\hline Partner died & 71 \\
\hline Religion & 28 \\
\hline Christianity & \multicolumn{2}{|l|}{} \\
\hline Islam & \multicolumn{2}{|l|}{} \\
\hline Traditional & Ethnicity & \\
\hline
\end{tabular}




\begin{tabular}{|l|l|l|}
\hline Hausa & 140 & 58.3 \\
\hline Yoruba & 27 & 11.3 \\
\hline Igbo & 73 & 30.4 \\
\hline Employment Status & \multicolumn{2}{|l|}{} \\
\hline Unemployed & 47 & 19.6 \\
\hline Self-Employed & 88 & 36.7 \\
\hline Employed & 36 & 15.0 \\
\hline Dependents & 37 & 15.4 \\
\hline Full time housewife & 32 & 13.3 \\
\hline Total & $\mathbf{2 4 0}$ & $\mathbf{1 0 0 . 0}$ \\
\hline
\end{tabular}

The female constitutes a higher number of respondents in the study, representing 176 (73.3\%). Most respondents, 89 (37.1\%), had secondary school level education and married 105 (43.8\%). The age group of 30-39 years was most dominant among the study population (38.3\%), followed by the age group 20-29 years $(30.8 \%)$. On respondents' religion, the majority were Christians 141 (58.8\%), followed by Moslem's $71(29.6 \%)$ and traditionalists 28 (11.7\%). On ethnicity, Hausas constituted the bulk of the respondents 140 (58.3\%), then Igbos 73 (30.4\%) and Yoruba's 27 (11.3\%) was the least among the study population. Employment status showed that a high percentage of respondents, $88(36.7 \%)$, were self-employed.

Table 2. Factors Affecting Retention of PLHIV in Care in the Study Site

\begin{tabular}{|c|c|c|c|c|}
\hline \multirow[t]{2}{*}{ Factors } & \multicolumn{4}{|c|}{ Percentage of respondents score $(n=240)$} \\
\hline & 1 & 2 & 3 & 4 \\
\hline \multicolumn{5}{|l|}{ Patient Factors } \\
\hline ARVs side effect & $137(57.1)$ & $40(16.7)$ & $37(15.4)$ & $26(10.8)$ \\
\hline Weight increase as a sign of wellness & $146(60.8)$ & $25(10.4)$ & $50(20.8)$ & $19(7.9)$ \\
\hline Alcohol use & $182(75.8)$ & $5(2.1)$ & $7(2.9)$ & $46(19.2)$ \\
\hline Religious/faith healing & 195(81.3) & $6(2.5)$ & $7(2.9)$ & $32(13.3)$ \\
\hline Use of other herbal remedies & $228(95.0)$ & $5(2.1)$ & $5(2.1)$ & $2(0.8)$ \\
\hline \multicolumn{5}{|l|}{ Social factor } \\
\hline Stigma and discrimination & $139(57.9)$ & $29(12.1)$ & $39(16.3)$ & $33(13.8)$ \\
\hline Use of herbal remedies & $215(89.6)$ & $6(2.5)$ & $11(4.6)$ & $8(3.3)$ \\
\hline Social support from family members & $172(71.7)$ & $27(11.3)$ & 23(9.6) & $18(7.5)$ \\
\hline Fear of marriage breakdown & $214(89.2)$ & $4(1.7)$ & $0(0.0)$ & $22(9.2)$ \\
\hline \multicolumn{5}{|l|}{ Economic Factor } \\
\hline Food insecurity & $159(66.3)$ & 19(7.9) & $37(15.4)$ & $25(10.4)$ \\
\hline Traveling on duty transportation of health facility & $184(76.7)$ & $18(7.5)$ & $27(11.3)$ & $11(4.6)$ \\
\hline Lack of employment & $177(73.8)$ & $5(2.1)$ & $10(4.2)$ & $48(20.0)$ \\
\hline \multicolumn{5}{|l|}{ Institutional related factors } \\
\hline Long waiting & $28(11.7)$ & 13(5.4) & $51(21.3)$ & $148(61.7)$ \\
\hline Stock of out of ARVs & $220(91.7)$ & $2(0.8)$ & $10(4.2)$ & $8(3.3)$ \\
\hline High patient load & $46(19.2)$ & $11(4.6)$ & $64(26.7)$ & 119(46.9) \\
\hline Travel distance to ART centres/transportation & $135(56.3)$ & $24(10.0)$ & $47(19.6)$ & $34(14.2)$ \\
\hline Inadequate space for ART clinic & $50(20.8)$ & $8(3.3)$ & $66(27.5)$ & $116(48.3)$ \\
\hline Inadequate health workers/staff & $89(37.1)$ & $9(3.8)$ & $67(27.9)$ & $75(31.3)$ \\
\hline
\end{tabular}




\begin{tabular}{|l|l|l|l|l|}
\hline Poor quality of services & $82(34.2)$ & $12(5.0)$ & $48(20.0)$ & $98(40.8)$ \\
\hline Difficulty in accessing health facility & $99(41.3)$ & $8(3.3)$ & $54(22.5)$ & $79(32.9)$ \\
\hline
\end{tabular}

1=Not at all; $2=$ Mild; $3=$ Moderate and 4= Severe

Of the 240 respondents interviewed, the main factor affecting their retention in care was institutional factor such as long waiting time (61.7\%), high patient load (46.9\%), Inadequate

space for ART clinic (48.3\%), Poor quality of services (40.8\%), difficulty in accessing health facility $(32.9 \%)$ and inadequate health workers/staff $(31.3 \%)$.

Table 3. Association Between Socio-demographic Characteristics of Respondents and Institutional Related Factors Affecting Retention

\begin{tabular}{|c|c|c|c|c|}
\hline \multirow[t]{3}{*}{ Characteristics } & \multicolumn{3}{|c|}{\begin{tabular}{|l|l} 
Institutional related factors & $\mathrm{X}^{2}$
\end{tabular}} & \multirow[t]{3}{*}{ P-value } \\
\hline & Not affected & Affected & & \\
\hline & $\mathbf{N}(\%)$ & $\mathbf{N}(\%)$ & & \\
\hline \multicolumn{5}{|l|}{ Age } \\
\hline $20-29$ & $48(64.9)$ & $26(35.1)$ & 3.560 & \multirow[t]{5}{*}{0.469} \\
\hline $30-39$ & $54(58.7)$ & $38(41.3)$ & & \\
\hline $40-49$ & $34(59.6)$ & $23(40.4)$ & & \\
\hline $50-59$ & $9(69.2)$ & $4(30.8)$ & & \\
\hline $60-69$ & $4(100.0)$ & $0(0.0)$ & & \\
\hline \multicolumn{5}{|l|}{ Sex } \\
\hline Male & $47(73.4)$ & $17(26.6)$ & 4.780 & \multirow[t]{2}{*}{0.290} \\
\hline Female & $102(58.0)$ & $74(42.0)$ & & \\
\hline \multicolumn{5}{|l|}{ Education } \\
\hline No formal education & $21(56.8)$ & $16(43.2)$ & 0.597 & \multirow[t]{4}{*}{0.897} \\
\hline Primary & $26(61.9)$ & $16(38.1)$ & & \\
\hline Secondary & $57(64.0)$ & $32(36.0)$ & & \\
\hline Tertiary & $45(62.5)$ & $27(37.5)$ & & \\
\hline \multicolumn{5}{|l|}{ Marital status } \\
\hline Never married & $70(74.5)$ & $24(25.5)$ & 18.225 & \multirow[t]{4}{*}{0.000} \\
\hline Married & $59(56.2)$ & $46(43.8)$ & & \\
\hline Separated/Divorced & $14(70.0)$ & $6(30.0)$ & & \\
\hline Partner died & $6(28.6)$ & $15(71.4)$ & & \\
\hline \multicolumn{5}{|l|}{ Religion } \\
\hline Christianity & $76(53.9)$ & $65(46.1)$ & 10.110 & \multirow[t]{3}{*}{0.006} \\
\hline Islam & $51(71.8)$ & $20(28.2)$ & & \\
\hline Traditional & $22(78.6)$ & $6(21.4)$ & & \\
\hline \multicolumn{5}{|l|}{ Ethnicity } \\
\hline Hausa & $84(60.0)$ & $56(40.0)$ & 1.073 & \multirow[t]{3}{*}{0.585} \\
\hline Yoruba & $19(70.4)$ & $8(29.6)$ & & \\
\hline Igbo & $46(63.0)$ & $27(37.0)$ & & \\
\hline \multicolumn{5}{|l|}{ Employment status } \\
\hline Unemployed & $19(40.4)$ & $28(59.6)$ & 13.603 & \multirow[t]{3}{*}{0.009} \\
\hline Self-Employed & $63(71.6)$ & $25(28.4)$ & & \\
\hline Employed & $21(58.3)$ & $15(41.7)$ & & \\
\hline
\end{tabular}




\begin{tabular}{|l|l|l|l|l|}
\hline Dependents & $25(67.6)$ & $12(32.4)$ & & \\
\cline { 1 - 3 } Full time housewife & $21(65.6)$ & $11(34.4)$ & & \\
\cline { 1 - 3 } Family & $40(65.6)$ & $21(34.4)$ & 5.486 & 0.139 \\
\cline { 1 - 3 } Living alone & $53(57.6)$ & $39(42.4)$ & & \\
\cline { 1 - 4 } Nuclear & $52(68.4)$ & $24(31.6)$ & & \\
\cline { 1 - 4 } Extended & $4(36.4)$ & $7(63.6)$ & & \\
\hline Cohabiting & $\mathbf{1 4 9 ( 6 2 . 1 )}$ & $\mathbf{9 1 ( 3 7 . 9 )}$ & & \\
\hline Total & & & & \\
\hline
\end{tabular}

*Fisher's exact test, significant at $\mathrm{p}<0.05$

A review of socio-demographic characteristics of respondents and institutional related factors affecting retention in care showed an association between marital status $(\mathrm{p}=0.000)$, religion $(\mathrm{p}=0.006)$, employment status $(\mathrm{p}=0.009)$, and institutional related factors affecting retention in care.

\section{Discussion}

This study sought to explore institutional factors that affect retention of PLHIV on treatment in Wuse General Hospital. In this comprehensive analysis of institutional factors that were associated with LTFU, it was observed that long waiting time, high patient load, stock out of ARV's, travel distance and transportation, inadequate space for ART clinic, inadequate health workers/staff, poor quality of services and difficulty in accessing health were institutional related factors affecting PLHIV retention in care.

Based on the findings of this study, it was observed that long waiting time was the most prominent factor affecting PLHIV retention. The long waiting time in the clinic contributed to poor retention because patients are frustrated and tired of waiting to be seen by healthcare providers. A similar study reported that long queuing duration was associated with LTFU among HIV-positive adults who attend care and treatment clinics [25]. Long waiting hours are one of the major drivers of attrition for patients on $\operatorname{ART}[26,27]$. Other findings from previous studies reported patients perceive long waiting hours at health facilities as a challenge to access service [28].
High patient load is another major factor affecting PLHIV retention from this study due to many reasons. The analysis of the findings shows that inadequate health workers /staff as a factor influencing retention in the care of patients on ART. This is because inadequate manpower may lead to long queues at the clinic waiting for the area and, consequently, long waiting hours. The shortage of human resources for health has severely hampered the rollout of ART in Sub-Saharan Africa [29]. Other studies have shown that decentralization of HIV services is associated with improved retention $[22,30]$ as care is brought closer to the homes of PLHIV. Another strategy that mitigates the long waiting time due to high patient load time is a task-shifting strategy from clinicians to lower cadres of health workers to increase access, cost-effectiveness in care, and retention of more patients in ART [29]. The promotion of a differentiated model of care and follow-up [31] with less frequent visits for clinically stable PLHIV who are virally suppressed will reduce the challenges of high patient load during clinic hours.

From the study, inadequate space for ART clinics was another challenge affecting PLHIV retention. This may be partly due to the congestion of patients in the waiting area, which may lead to a lack of confidentiality (privacy) during ART clinics because of overcrowding of patients. Previous studies revealed that some patients might shun appointments where there is no confidentiality and can lead to lost to fellow up [32]. 
Poor quality of services was reported by PLHIV is one of the drivers of poor retention of patients in care in Wuse General Hospital; reasons may be due to shortage of health personnel at the facility. Previous reports have shown that many public facilities in subSaharan Africa scaled up without a comparable increase in the number of healthcare personnel to attend to the larger number of patients [33]. The high patient load at health facilities may also result in decrease patient-provider interaction and poor quality of care due to work pressure leading to overall PLHIV dissatisfaction with the quality of care being offered. PLHIV believed that the burden and strain on the health care system due to high patient load and the exhaustion experienced by healthcare workers would lead to poor service delivery, which could have been averted if the facility has adequate manpower.

From the analysis of this study, some patients were affected by travel distance to ART centres/transportation. Previous studies reported that travel distance to ART centers was a barrier to accessing treatment, and some mentioned transportation cost as a reason for defaulting. A similar study revealed that a substantial proportion of participants that lived far from the clinic $(31 \%>50 \mathrm{~km})$ was associated with a higher risk of LTFU [34]. Accessibility problems such as lack of transport, cost, or distance have previously been identified as barriers to clinic attendance. Travel distance to clinic sites and associated costs have been described as obstacles to retention [20] and probably a reason for LFTU.

Potential interventions that could improve retention of PLHIV in care include longer ART refills for those stable on treatment $[34,35]$ and decentralization of care to local health clinics, or alternative ART delivery models such as taking ARVs to the home or going through the community [36] will help to decongest comprehensive ART sites. Some individuals may prefer services closer to their homes to minimize travelling, and others may purposely avoid clinics close to their homes in order to avoid stigma from community members [37].

\section{Conclusion}

Despite free ART services being offered in the HIV clinics at Wuse General Hospital, institutional factors pose challenges to PLHIV retention in care. Long waiting time, high patient load, stock out of ARV's, travel distance and transportation fare, inadequate space for ART clinic, inadequate health workers/staff, poor quality of services (inadequate manpower, poor laboratory services, drug stock out, long waiting clinic hours, etc) and difficulty in accessing health care were institutional related factors found to affect retention of PLHIV in care. Interventions that will address these factors could improve PLHIV retention on ART. For institutional factors such as travel distance to health facilities and high transport cost, patients should be continually encouraged to access treatment at their nearest treatment centre based on their choice. Strengthening the decentralization of HIV services so that stable patient can pick their drug refill at the facility nearest to them rather than travelling long distances to ART centres that will cost them so much should be considered. Other interventions that could improve PLHIV retention in ART include strengthening the task shifting policy, the government employing more staff, and increasing the number of clinic days in a week to reduce the long waiting hours.

\section{Conflict of Interest}

There is no conflict of interest in this study.

\section{Acknowledgments}

I would like to express my gratitude to all who have helped me to accomplish this thesis and my study at Wuse General Hospital Abuja. First and foremost, I would like to thank Professor Musa Babashani, my supervisor, for his interest in this study, excellent ideas, and his dedication to reading and providing comments on multiple drafts of my thesis. He also 
encouraged and guided me to be able to steer my academic study and professional development. I would like to express my gratitude to my wife Dr. Belinda Uba, for her efforts and encouragement in this thesis. I also

\section{References}

[1] Mondal, M., and Shitan, M., 2013, Factors affecting the HIV/AIDS epidemic: an ecological analysis of global data. Afr Health Sci, 13(2):301310.

[2] Health FM, 2010, National guidelines for HIV and AIDS treatment and care in adolescents and adults. Federal Ministry of Health Abuja.

[3] Mberi, M.N., Kuonza, L.R., Dube, N.M., Nattey, C., Manda, S., and Summers, R., 2015, Determinants of loss to follow-up in patients on antiretroviral treatment, South Africa, 2004-2012: a cohort study. BMC Health Serv Res, 15(1):1-11.

[4] WHO. 13-15 September 2011. 2012, Retention in HIV programmes: defining the challenges and identifying solutions: meeting report.

[5] Fuente-Soro, L., López-Varela E., Augusto, O., Bernardo, E.L., Sacoor, C., and Nhacolo, A., 2020, Loss to follow-up and opportunities for reengagement in HIV care in rural Mozambique: A prospective cohort study. Medicine (Baltimore), 99(20): e20236.

[6] Cohen, M.S., Chen, Y.Q., McCauley, M., Gamble, T., Hosseinipour, M.C., and Kumarasamy, N., 2011, Prevention of HIV-1 Infection with Early Antiretroviral Therapy. N Engl J Med, 365(6):493505.

[7] Fox MP and Rosen, S., 2010, Patient retention in antiretroviral therapy programs up to three years on treatment in Sub-Saharan Africa, 2007-2009: systematic review. Trop Med Int Health, 15:1-15.

[8] Babatunde, O., Ojo, O.J., Atoyebi, O.A., Ekpo, D.S., Ogundana, A.O., and Olaniyan, T.O., 2015, Seven-year review of retention in HIV care and treatment in federal medical centre Ido-Ekiti. Pan Afr Med J, 22(1).

[9] Amuron, B., Namara, G., Birungi, J., Nabiryo, C., Levin, J., and Grosskurth, H., 2009, Mortality and loss-to-follow-up during the pre-treatment recognize and appreciate the wonderful work of Dr. Adedayo Adeyemi, Mr. Thaddeus Olatunbosun, and field assistance that, despite their busy schedule, helped me to accomplish this work.

period in an antiretroviral therapy programme under normal health service conditions in Uganda. $B M C$ Public Health, 9(1):290.

[10] Giordano, T.P., Gifford, A.L., White Jr, A.C., Almazor, MES., Rabeneck, L., and Hartman C, 2007, Retention in care: a challenge to survival with HIV infection. Clin Infect Dis, 44(11):1493-1499.

[11]Paracha, S.I., Hafeez, F., Habib, H., Yaqoob, A., Fatima, R., and Saeed, Q., 2019, The extent of loss to follow among patients initiated on antiretroviral treatment, Pakistan 2017-2018: a retrospective cross-sectional study.

[12]Zürcher, K., Mooser, A., Anderegg, N., Tymejczyk, O., Couvillon, M.J., and Nash, D., 2017, Outcomes of HIV-positive patients lost to follow-up in African treatment programmes. Trop Med Int Health., 22(4):375-387.

[13]Egger, M., Spycher, B.D., Sidle, J., Weigel, R., Geng, E.H., and Fox, M.P., 2011. Correcting mortality for loss to follow-up: a nomogram applied to antiretroviral treatment programmes in subSaharan Africa. PLoS Med.; 8(1): e1000390.

[14] Cornell, M., Lessells, R., Fox, M.P., GARONE, D.B, Giddy, J., and Fenner, L., 1999. Mortality among adults transferred and lost to follow-up from antiretroviral therapy programmes in South Africa: a multicentre cohort study. J Acquir Immune Defic Syndr. 2014;67(2): e67.

[15] Geng, E.H., Odeny, T.A., Lyamuya, R., Nakiwogga-Muwanga. A., Diero, L., and Bwana, M., 2016, Retention in care and patient-reported reasons for undocumented transfer or stopping care among HIV-infected patients on antiretroviral therapy in Eastern Africa: application of a samplingbased approach. Clin Infect Dis. 62(7):935-944.

[16] Brinkhof, M.W., Pujades-Rodriguez, M., and Egger M., 2009, Mortality of patients lost to followup in antiretroviral treatment programmes in 
resource-limited settings: systematic review and meta-analysis. PloS One, 4(6): e5790.

[17] Opio, D., Semitala, F.C., Kakeeto, A., Sendaula E., Okimat, P., and Nakafeero, B., 2019, Loss to follow-up and associated factors among adult people living with HIV at public health facilities in Wakiso district, Uganda: a retrospective cohort study. $B M C$ Health Serv Res; 19(1):628.

[18] Rachlis, B., Bakoyannis, G., Easterbrook, P., Genberg, B., Braithwaite, R.S., and Cohen C.R., 2016, Facility-level factors influencing retention of patients in HIV care in East Africa. PloS One, 11(8): e0159994.

[19] Geng, E.H., Bangsberg, D.R., Musinguzi, N., Emenyonu, N., Bwana, M.B., and Yiannoutsos, C.T.., 2010, Understanding reasons for and outcomes of patients lost to follow-up in antiretroviral therapy programs in Africa through a sampling-based approach. J Acquir Immune Defic Syndr, 53(3):405.

[20]Tuller, D.M., Bangsberg D.R., Senkungu, J., Ware N.C., Emenyonu, N., and Weiser SD., 2010, Transportation costs impede sustained adherence and access to HAART in a clinic population in southwestern Uganda: a qualitative study. AIDS Behav, 14(4):778-784.

[21] Ochieng-Ooko, V., Ochieng, D., Sidle, J.E., Holdsworth, M., Wools-Kaloustian, K., and Siika, A.M., 2010, Influence of gender on loss to follow-up in a large HIV treatment programme in western Kenya. Bull World Health Organ, 88:681-688.

[22] Chan A.K, Mateyu, G., Jahn, A., Schouten, E., Arora, P., Mlotha, W., 2010, Outcome assessment of decentralization of antiretroviral therapy provision in a rural district of Malawi using an integrated primary care model. Trop Med Int Health, 15:90-97. [23] Dalhatu I, Onotu D, Odafe S, Abiri O, Debem H, Agolory S, Shiraishi RW, Auld AF, Swaminathan M, Dokubo K, Ngige E, Asadu C, Abatta E, Ellerbrock., 2016, Outcomes of Nigeria's HIV/AIDS Treatment Program for Patients Initiated on Antiretroviral Treatment between 2004-2012.

[24] Assefa Y, Worku A, Wouters E, Koole O, Haile Mariam D, Van Damme W. 2012, Simplified tools for measuring retention in care in antiretroviral treatment program in Ethiopia: cohort and current retention in care. PLoS One. 2012;7(6): e38555. doi: 10.1371/journal.pone.0038555.

[25]Chemutai, R.J, Mwaniki, P., Mutai, J.K., Njenga, S., and Kyalo, M.A., 2018, Factors Associated with Retention in Care among HIV Positive Adults Attending Pumwani Comprehensive Care Centre, In Nairobi County, Kenya.

[26]Bekker, L.G., Venter, F., Cohen, K., Goemare,

E., Van Cutsem, G., and Boulle, A., 2014, Provision of antiretroviral therapy in South Africa: the nuts and bolts.

[27] Miller, C.M., Ketlhapile, M., Rybasack-Smith, H., and Rosen, S., 2010, Why are antiretroviral treatment patients lost to follow-up? A qualitative study from South Africa. Trop Med Int Health; $15: 48-54$.

[28]Fernandes, C.M., Daya, M.R., Barry. S., and Palmer, N., 1994, Emergency department patients who leave without seeing a physician: the Toronto Hospital experience. Ann Emerg Med, 24(6):10921096.

[29] Callaghan, M., Ford, N., and Schneider, H., 2010, A systematic review of task-shifting for HIV treatment and care in Africa. Hum Resour Health; 8(1):8.

[30] Govindasamy, D., Ford, N, and Kranzer, K.., 2012, Risk factors, barriers and facilitators for linkage to antiretroviral therapy care 2012: a systematic review. Aids, 26 16):2059-2067.

[31] Duncombe, C., Rosenblum, S., Hellmann, N., Holmes, C., Wilkinson, L., and Biot, M., 2015, Reframing HIV care: putting people at the centre of antiretroviral delivery. Trop Med Int Health, 20(4):430-447.

[32] Mukumbang, F.C., Mwale, J.C., and Van Wyk, B., 2017, Conceptualising the factors affecting retention in care of patients on antiretroviral treatment in Kabwe District, Zambia, using the ecological framework. AIDS Res Treat.

[33] Mwale, J.C., 2016, Factors affecting retention in care of patients on antiretroviral treatment in the Kabwe district, Zambia.

[34] Kalinjuma, A.V., Glass, T.R., Weisser, M., Myeya, S.J, Kasuga, B., and Kisung'a, Y., 2020, Prospective assessment of loss to follow-up: incidence and associated factors in a cohort of HIV- 
positive adults in rural Tanzania. J Int AIDS Soc, 23(3): e25460.

[35]Larson, B.A., Brennan, A., McNamara, L., Long, L., Rosen, S., and Sanne, I., 2010, Early loss to follow up after enrolment in pre-ART care at a large public clinic in Johannesburg, South Africa. Trop Med Int Health, 15:43-47.

[36] Francis, J., Geldsetzer, P., Ulenga, N., Sando, D., Lema, I., and Mboggo, E., 2018, The impact of community delivery of antiretroviral therapy on viral load suppression: findings from a pragmatic randomized non-inferiority trial in Dar es Salaam, Tanzania. In: Journal of the International AIDS Society. John Wiley \& Sons Ltd the atrium, southern gate, chichester, PO19 8SQ, W 94-94.

[37]Layer, E.H., Kennedy, C.E., Beckham, S.W., Mbwambo, J.K, Likindikoki, S., Davis, W.W., 2014, Multi-level factors affecting entry into and engagement in the HIV continuum of care in Iringa, Tanzania. PloS One, 9(8): e104961. 